Service social

\title{
Agir contre la violence, par Ginette Larouche, Montréal, Les éditions de La Pleine Lune, 1987, 550 pages.
}

\section{Lorraine Fleury}

Volume 37, numéro 1-2, 1988

Par-delà les barrières des sexes

URI : https://id.erudit.org/iderudit/706403ar

DOI : https://doi.org/10.7202/706403ar

Aller au sommaire du numéro

Éditeur(s)

École de service social de l'Université Laval

ISSN

1708-1734 (numérique)

Découvrir la revue

Citer ce compte rendu

Fleury, L. (1988). Compte rendu de [Agir contre la violence, par Ginette Larouche, Montréal, Les éditions de La Pleine Lune, 1987, 550 pages.] Service social, 37(1-2), 282-284. https://doi.org/10.7202/706403ar d'utilisation que vous pouvez consulter en ligne.

https://apropos.erudit.org/fr/usagers/politique-dutilisation/ 
Enfin, est-ce une analyse objective des rapports entre les hommes et les femmes que Farrell nous présente ici, ou ne veut-il pas plutôt, et de façon très consciente, montrer l'autre côté de la médaille, c'est-à-dire le vécu des hommes dans cette période de remise en question des rôles sexuels? Malgré cette limitation, ou peut-être grâce à elle, ce livre représente une lecture extrêmement stimulante pour toute personne qui veut réfléchir sur la condition masculine d'aujourd'hui.

Thomas ANTIL

Agent de recherche,

Direction de la planification et de l'évolution,

Ministère de la santé et des services sociaux.

Agir contre la violence, par Ginette LAROUCHE, Montréal, Les éditions de La Pleine Lune, 1987, 550 pages.

Agir contre la violence présente une option féministe à l'intervention auprès des femmes qui ont subi de la violence en milieu conjugal. Cette violence peut se rapporter à de multiples formes d'agressions : physiques, psychologiques, verbales ou sexuelles. Délaissant l'approche familiale, Ginette Larouche a choisi d'aider les femmes violentées à exploiter leurs capacités et leurs compétences afin qu'elles puissent assurer leur protection et augmenter leur qualité de vie en "se choisissant " d'abord elles-mêmes.

Au Québec, Ginette Larouche fait figure de pionnière en matière de violence conjugale, et il est évident que ce livre s'appuie sur une solide expérience de praticienne et de formatrice. Son souci constant d'allier la théorie à la pratique fait de cet ouvrage un outil de travail bien documenté, agréable à lire, foisonnant de techniques de soutien et d'exercices utilisables, soit en entrevue individuelle, soit en groupe. L'auteure n'en est d'ailleurs pas à ses premières armes sur le sujet. Au début du livre, l'historique du modèle proposé en fait foi. Mentionnons aussi que Ginette Larouche a décrit son modèle d'intervention dans le Guide d'intervention auprès des femmes violentées publié en 1985 par la Corporation professionnelle des travailleurs sociaux du Québec. Le présent ouvrage est cependant beaucoup plus étoffé.

Dans l'avant-propos et le premier chapitre, portant sur les notions de base, l'auteure situe bien la violence dans son contexte socio-politique. "La violence, écrit-elle, est la forme ultime de l'oppression d'un groupe par un autre ou d'un sexe par l'autre." L'analyse féministe qui sous-tend son intervention relie les difficultés des femmes aux racines sociales de l'inégalité des sexes. Cette lecture de la réalité ne confond pas les conséquences de la violence avec la personnalité de la victime. La dépression, par exemple, est identifiée comme l'une des conséquences du vécu de violence et non comme une caractéristique de la femme battue. 
Le deuxième chapitre est consacré à l'entrevue en situation de crise, qui a pour objectifs principaux d'abaisser les tensions émotives, favoriser une prise de décision et offrir de l'aide concrète à la femme victime de violence. Le fait qu'il est difficile de se retrouver dans les subdivisions de ce chapitre est heureusement compensé par des tableaux synthétiques, que l'on retrouve d'ailleurs à la fin de chacun des chapitres.

L'auteure porte ensuite notre attention sur la restauration de l'estime de soi. J'ai beaucoup apprécié la technique du cœur, l'échange sur la symbiose du couple... Je suis certaine que toute intervenante pourra y puiser des outils adaptés à sa propre façon d'intervenir. Pour moi, il est d'ailleurs essentiel d'utiliser ce livre avec imagination, en l'adaptant à soi, à son contexte institutionnel et avant tout aux besoins spécifiques de chacune des femmes victimes de violence. Il est à ce titre très inspirant et stimulant.

Le quatrième chapitre, sur l'affirmation de soi, trouve un prolongement très intéressant au chapitre suivant qui traite de la colère. En effet, Ginette Larouche nous propose différentes façons de réapprivoiser ce sentiment trop souvent banni du répertoire émotif des femmes. Elle suggère des exercices pour exprimer la colère de façon affirmative et elle nous amène à ne pas confondre celle-ci et l'agression.

L'auteure aborde ensuite les dimensions multiples de la violence conjugale : dépistage, vécu des enfants, traitement de l'agresseur. Les pistes d'intervention sont concrètes et tiennent compte de nos réalités de pratique actuelles, celle par exemple du double mandat de protection de l'enfant et d'intervention auprès de la famille.

Le septième chapitre porte sur une stratégie essentielle de l'approche féministe : l'intervention de groupe. Il se termine d'ailleurs, à titre d'illustration, par le cheminement d'un groupe de femmes victimes de violence. L'auteure consacre également quelques pages à des situations qui attestent d'un malaise dans un groupe et à la façon de les éclaircir. Pour soutenir le modèle de pratique qu'elle met de l'avant, Ginette Larouche n'a pas ménagé les évaluations et les tests, mesurant entre autres l'augmentation de l'estime de soi, des capacités affirmatives et les changements dans le couple; il me semble cependant quelque peu fastidieux d'administrer tous ces tests avant et après un groupe. II reste que cela peut s'avérer utile dans la mesure où la marge de manœuvre dans l'institution est réduite et où la pertinence de l'intervention a besoin d'être démontrée.

Le dernier chapitre présente d'abord un texte sur le vécu des intervenantes impliquées dans le programme de formation et rédigé par Ann Pâquet-Deehy; un second, écrit par Maryse Rinfret-Raynor, porte sur la pratique et la recherche. Cette dernière partie $m$ 'a laissée sur mon appétit. Une recherche évaluative, qui se terminera en 1989, a été entreprise pour comparer les effets du traitement psychosocial reçu par trois groupes de femmes victimes de violence conjugale : un premier groupe, bénéficiant d'une thérapie de groupe, selon le modèle de l'intervention féministe; un deuxième, d'une thérapie individuelle, selon le modèle d'intervention féministe ; et un troisième, d'une thérapie selon l'approche habituelle des services sociaux. Les intervenantes des deux premiers groupes ont 
suivi la formation avec Ginette Larouche à l'Université de Montréal. J'ose espérer que les résultats seront publiés.

Ce livre nous laisse donc dans l'espérance: attente des résultats de la recherche mentionnée, mais surtout espoir que cesse la violence grâce à des actions concertées du réseau de l'État et du réseau communautaire ; grâce aussi à de véritables changements dans les rapports entre les hommes et les femmes.

Ensemble, nous pouvons faire des pas vers de nouvelles réalités où tous les rapports sociaux seraient égalitaires. C'est, pour l'instant, cet idéal jamais atteint qui nourrit ma pratique et qu'un livre comme celui de Ginette Larouche laisse mieux entrevoir.

Lorraine FLEURY

C.L.S.C. Lotbinière-Ouest.

Handbook of Counseling and Psychotherapy with Men, par M. SCHER, M. STEVENS, G. Good et G.A. Eichenfield (éds.), Newbury Park (Cal.), Sage, 1987, 400 pages.

En quoi la relation d'aide est-elle différente lorsqu'elle s'adresse aux hommes ? Quelles sont les meilleures techniques à utiliser avec les homosexuels ou les hommes atteints du SIDA ? Est-ce que l'âge d'un client masculin affecte l'approche utilisée en counseling ? Pères célibataires et difficultés d'adaptation, carrière et estime de soi, intervention auprès des " hommes batteurs ", counseling avec des hommes physiquement handicapés, avec des hommes en prison, telles sont quelques questions et problématiques auxquelles les vingt-six chapitres de ce livre tentent d'apporter un éclairage conceptuel et pratique.

Avant d'aborder toutes ces questions toutefois, le volume débute avec un chapitre de Pleck, où celui-ci rappelle quelques vicissitudes de la libération masculine des quinze dernières années : l'augmentation du temps investi par les hommes dans leurs rôles familiaux en même temps que l'augmentation, pour plusieurs, d'un inconfort psychologique face aux femmes, les attentes et ancrages persistants face aux rôles traditionnels versus le désir et la nécessité de changement vers de nouvelles formes de masculinité. Cette introduction est en quelque sorte le canevas de base pour comprendre les conflits psychosociaux vécus par les hommes d'aujourd'hui.

La première partie comprend huit chapitres. Différents auteurs dégagent plusieurs points de vue et techniques portant sur : le counseling de groupe pour les hommes et ses aspects particuliers, le counseling sur les problèmes reliés au monde du travail, le counseling sexuel ou le counseling de deuil. La deuxième partie regroupe ensuite trois chapitres dans une perspective typiquement développementale et discute de l'intégration de l'identité chez les adolescents et des enjeux développementaux propres aux hommes âgés. La troisième partie, "Ethnicité", se penche sur les problèmes spécifiques de counseling avec les hommes des communautés asiatiques, noires ou hispaniques. Préjugés culturels, 